\title{
Confinement, deconfinement and chiral symmetry breaking in QCD
}

\section{Yuri Simonov*}

Institute of Theoretical and Experimental Physics

E-mail: simonov@itep.ru

Confinement is ensured by quadratic colorelectric field correlators of special type, denoted by $D^{E}(x)$. The latter together with four other quadratic correlators, $D^{H}, D_{1}^{E}, D_{1}^{H}$ and $D^{E H}$ support most of perturbative and nonperturbative dynamics, while contribution of higher order terms is shown to be small. These correlators, on the other hand, can be calculated via gluelump Green's function, where dynamics is defined by the correlators themselves. In this way one obtains a self-consistent scheme, where string tension can be expressed in terms of $\Lambda_{Q C D}$. This confinement mechanism ensures also chiral symmetry breaking and disappears at $T \geq T_{c}$ where $D^{E}(x)$ vanishes.

International Workshop on QCD Green?s Functions, Confinement and Phenomenology

September 7-11, 2009

ECT Trento, Italy

\footnotetext{
${ }^{*}$ Speaker.
} 


\section{Introduction}

Confinement is the most important property of the QCD vacuum (see [1, 2] for reviews) and as shown on the lattice and analytically it can be quite naturally explained as arising from properties of quantum stochastic ensemble of nonperturbative $(n p)$ fields filling QCD vacuum. The development of this approach in a systematic way started in 1987 [3] (see, e.g. [4] as example of earlier investigations concerning stochasticity of QCD vacuum). The nontrivial structure of $n p$ vacuum can be described by a set of nonlocal gauge-invariant field strength correlators (FC). We discuss this scenario in the present talk (see [5] for an extended version of some results of this talk).

QCD sum rules [6] were suggested as an independent approach to $n p Q C D$ dynamics, not addressing directly confinement mechanism. The key role is played by gluon condensate $G_{2}$, which is defined as $n p$ average of the following type:

$$
G_{2}=\frac{\alpha_{s}}{\pi}\left\langle F_{\mu v}^{a}(0) F_{\mu v}^{a}(0)\right\rangle
$$

However the relation of $G_{2}$ to confinement is rather tricky, since $G_{2}$ is not an order parameter and, in particular, neither perturbative nor $n p$ contributions to this quantity vanish in either phase. On the other hand one can show (see details in [7]) that the scale of deconfinement temperature is set by the condensate, or, more precisely, by its electric part: $T_{c} \sim G_{2}^{1 / 4}$. One clearly sees some tension between this "small" scale and a "large" mass scale in QCD given by, e.g. the mass of the lightest $0^{++}$glueball, which is about $1.5 \mathrm{GeV}$.

The question about the origin of this mismatch turned out to be rather deep. It was suggested in [3], that there is another important dimensionfull parameter characterizing $n p$ dynamics of vacuum fields: correlation length $\lambda$ (also denoted as $T_{g}$ in some papers). The crucial feature of stochastic picture found in [9] is that the lowest, quadratic, nonlocal FC, $\left\langle F_{\mu v}(x) F_{\lambda \sigma}(y)\right\rangle$ describes all $n p$ dynamics with very good accuracy. It was also shown that a simple exponential form of quadratic correlators found on the lattice [9] allows to calculate the correlation length $\lambda$ entering these exponents, and since $\lambda$ is small, potential relativistic quantum-mechanical picture is applicable and all QCD spectrum is defined mostly by string tension $\sigma$ (which is an integral characteristic of the nonlocal correlator, see below) and not by its exact profile (see $[3,10]$ for reviews of meson, glueball hybrid spectra).

However to establish the confinement mechanism unambiguously, one should be able to calculate vacuum field distributions, i.e. field correlators, self-consistently. In the long run it means that one is to demonstrate that it is essential property of QCD vacuum fields ensemble to be characterized by correlators, which support confinement for temperatures $T$ below some critical value $T_{c}$, and deconfinement at $T>T_{c}$.

The main aim of this talk is to present this set of equations as a self-consistent mechanism of confinement and to clarify qualitative details of the FC - gluelump connection.

\section{Wegner-Wilson loop, Field Correlators and Green's Functions}

The Wilson loop is the basic element for discussion of confinement (area law), but also it enters as a main dynamical kernel in the Fock-Feynman-Schwinger Representation (FFSR) of Green's functions of any white QCD object [11]. 
It is convenient to use nonabelian Stokes theorem (see [2] for references and discussion) for the Wilson loop) and to rewrite it as an integral over the surface $S$ inside the contour $C=C(x, y)$ :

$$
\begin{gathered}
\langle W(C)\rangle=\left\langle\operatorname{Tr} P \exp \left(i g \int_{C} A_{\mu}(z) d z_{\mu}\right)\right\rangle=\left\langle\operatorname{Tr} \mathscr{P} \exp \left(i g \int_{S} F(u) d s(u)\right)\right\rangle= \\
=\operatorname{Tr} \mathscr{P}_{x} \exp \sum_{n=2}^{\infty}(i g)^{n} \int_{S}\left\langle\left\langle F\left(u_{1}\right) . . F\left(u_{n}\right)\right\rangle\right\rangle d s_{1} . . d s_{n}=\exp \sum_{n=2}^{\infty} \Delta^{(n)}[S]
\end{gathered}
$$

The central role in the discussed method is played by quadratic (Gaussian) FC of the form

$$
D_{\mu v, \lambda \sigma} \equiv g^{2}\left\langle\operatorname{Tr} F_{\mu v}(x) \Phi(x, y) F_{\lambda \sigma}(y) \Phi(y, x)\right\rangle
$$

where $F_{\mu \nu}$ is the field strength and $\Phi(x, y)$ is the parallel transporter. Correlation lengths $\lambda_{i}$ for different channels are defined in terms of asymptotics of (2.2) at large distances: $\exp \left(-|x-y| / \lambda_{i}\right)$. The physical role of $\lambda_{i}$ is very important since it distinguishes two regimes: one expects validity of potential-type approach describing the structure of hadrons of spatial size $R$ and at temporal scale $T_{q}$ for $\lambda_{i} \ll R, T_{q}$, while in the opposite case, when $\lambda_{i} \gg R, T_{q}$ the nonpotential description in terms of spatially homogeneous condensates can be applied.

At zero temperature the $O(4)$ invariance of Euclidean space-time holds ${ }^{1}$ and FC (2.2) is represented through two scalar functions $D(z), D_{1}(z)$ (where $z \equiv x-y$ ) as follows

$$
\begin{gathered}
D_{\mu \nu, \lambda \sigma}(z)=\left(\delta_{\mu \lambda} \delta_{v \sigma}-\delta_{\mu \sigma} \delta_{v \lambda}\right) D(z)+\frac{1}{2}\left[\frac{\partial}{\partial z_{\mu}}\left(z_{\lambda} \delta_{v \sigma}-z_{\sigma} \delta_{v \lambda}\right)+\right. \\
\left.+\frac{\partial}{\partial z_{v}}\left(z_{\sigma} \delta_{\mu \lambda}-z_{\lambda} \delta_{\mu \sigma}\right)\right] D_{1}(z) .
\end{gathered}
$$

One has to distinguish from the very beginning perturbative and $n p$ parts of the correlators $D(z), D_{1}(z)$. Beginning with the former, one easily finds at tree level

$$
D^{p, 0}(z)=0 ; D_{1}^{p, 0}(z)=C_{2}(f) \frac{4 \alpha_{s}}{\pi} \frac{1}{z^{4}}
$$

where $C_{2}(f)$ is fundamental Casimir $C_{2}(f)=\left(N_{c}^{2}-1\right) / 2 N_{c}$. At higher orders situation becomes more complicated. Namely, one has at $n$-loop order

$$
D^{p, n}(z)=D_{1}^{p, 0}(z) \cdot G^{(n)}(z) ; D_{1}^{p, n}(z)=D_{1}^{p, 0}(z) \cdot G_{1}^{(n)}(z)
$$

where the gauge-invariant functions $G^{(n)}(z), G_{1}^{(n)}(z)$ has the following general structure:

$$
G^{(n)}(z), G_{1}^{(n)}(z) \sim \alpha_{s}^{n}\left[c_{n}(\log \mu z)^{n}+\ldots\right]
$$

Thus we assume (and confirm aposteriori) that the following decomposition takes place (vanishing of the perturbative part of $D(z)$ is shown in [12]

$$
D(z)=D^{n p}(z) ; D_{1}(z)=D_{1}^{p}(z)+D_{1}^{n p}(z)
$$

\footnotetext{
${ }^{1}$ All treatment in this paper as well as averaging over vacuum fields is done in the Euclidean space. Notice that only after all Green's functions are computed, analytic continuation to Minkowskii space-time can be accomplished.
} 
and $D(z)$ has a smooth limit when $z \rightarrow 0$. Notice that by $D_{1}(0)$ we always understand $n p$ "condensate" part in what follows:

$$
G_{2}=\frac{6 N_{c}}{\pi^{2}}\left(D^{n p}(0)+D_{1}^{n p}(0)\right)
$$

As is shown below, the correlation lengths $\lambda$ and $\lambda_{1}$ of $D(z)$ and $D_{1}^{n p}(z)$ respectively appear to be just inverse masses of the corresponding gluelumps $\lambda_{j}=1 / M_{j}$.

Having analytic expressions for FCs one might ask how to check them versus experimental and lattice data. On experimental side in hadron spectroscopy one measures masses and transition matrix elements, which are defined by dynamical equation and the latter can be used of potential type due to smallness of $\lambda_{j}$. In static potential only integrals over distance enter and the spinindependent static potential can be written as [3]

$$
\begin{aligned}
V_{Q \bar{Q}}(r)= & 2 \int_{0}^{r}(r-\lambda) d \lambda \int_{0}^{\infty} d v D^{E}\left(\sqrt{\lambda^{2}+v^{2}}\right)+ \\
& +\int_{0}^{r} \lambda d \lambda \int_{0}^{\infty} D_{1}^{E}\left(\sqrt{\lambda^{2}+v^{2}}\right) .
\end{aligned}
$$

Making use of (2.4) together with definition of the string tension [3],

$$
\sigma=\frac{1}{2} \iint d^{2} z D(z)
$$

one obtains from (2.8) the standard form of the static potential for $N_{c}=3$ at distances $r \gg \lambda$

$$
V_{Q \bar{Q}}(r)=\sigma r-\frac{4 \alpha_{s}(r)}{3 r}+\mathscr{O}\left(\lambda / r, \alpha_{s}^{2}\right)
$$

As is argued below, $\lambda \approx 0.1 \mathrm{Fm}$, so that the form (2.10) is applicable in the whole range of distances $r>0.1 \mathrm{fm}$ provided asymptotic freedom is taken into account in $\alpha_{s}(r)$ in (2.10).

\section{Gluelumps and FC}

In this section we establish a connection of FC $D(z)$ and $D_{1}(z)$ with the gluelump Green's functions.

To proceed one can use the background field formalism [13][14], where the notions of valence gluon field $a_{\mu}$ and background field $B_{\mu}$ are introduced, so that total gluonic field $A_{\mu}$ is written as

$$
A_{\mu}=a_{\mu}+B_{\mu}
$$

The main idea we are going to adopt here is suggested in [15].

Now we turn to the analytic calculation of FC in terms of gluelump Green's function. To this end we insert (3.1) into (2.2) and have for $F_{\mu v}(x)$ :

$$
F_{\mu v}(x)=\partial_{\mu} A_{v}-\partial_{v} A_{\mu}-i g\left[A_{\mu}, A_{v}\right]=\hat{D}_{\mu} a_{v}-\hat{D}_{v} a_{\mu}-i g\left[a_{\mu}, a_{v}\right]+F_{\mu v}^{(B)} .
$$

Here, the term $F_{\mu \nu}^{(B)}$ contains only the field $B_{\mu}^{b}$. It is clear that when one averages over field $a_{\mu}^{a}$ and sums finally over all color indices $a$, one actually exploits all the fields with color indices from 
$F_{\mu \nu}^{(B)}$, so that the term $F_{\mu v}^{(B)}$ can be omitted, if summing over all indices $a$ is presumed to be done at the end of calculation (see [15] for more discussion).

As a result $D_{\mu v, \lambda \sigma}$ can be written as

$$
D_{\mu v, \lambda \sigma}(x, y)=D_{\mu v, \lambda \sigma}^{(0)}+D_{\mu v, \lambda \sigma}^{(1)}+D_{\mu v, \lambda \sigma}^{(2)}
$$

where the superscript $0,1,2$ refers to the power of $g$ coming from the term $i g\left[a_{\mu}, a_{v}\right]$.

We can address now an important question about the relation between FCs and gluelump Green's functions. We begin with 1-gluon gluelump, whose Green's function reads

$$
G_{\mu \nu}^{(1 g)}(x, y)=\left\langle\operatorname{Tr}_{a} a_{\mu}(x) \hat{\Phi}(x, y) a_{v}(y)\right\rangle .
$$

As shown in [16], the first term in (3.5) is connected to the functions $D_{1}$ and it can be written as follows

$$
D_{\mu v, \lambda \sigma}^{(0)}(x, y)=\frac{g^{2}}{2 N_{c}^{2}}\left\{\frac{\partial}{\partial x_{\mu}} \frac{\partial}{\partial y_{\lambda}} G_{v \sigma}^{(1 g)}(x, y)+\text { perm }\right\}+\Delta_{\mu v, \lambda \sigma}^{(0)},
$$

where $\Delta_{\mu v, \lambda \sigma}^{(0)}$ contains contribution of higher FCs, which we systematically discard.

On the other hand one can find $G_{\mu \nu}^{(1 g)}(x, y)$ from the FFSR for $G_{\mu \nu}^{(1 g)}$ [11] expression [?] written as

$$
G_{\mu \nu}^{(1 g l)}(x, y)=\operatorname{Tr}_{a} \int_{0}^{\infty} d s(D z)_{x y} \exp (-K)\left\langle W_{\mu v}^{F}\left(C_{x y}\right)\right\rangle,
$$

where the spin-dependent $\mathrm{W}$-loop is

$$
W_{\mu v}^{F}\left(C_{x y}\right)=P P_{F}\left\{\exp \left(i g \int B_{\lambda} d z_{\lambda}\right) \exp F\right\}_{\mu \nu}
$$

and the gluon spin factor is $\exp F \equiv \exp \left(2 i g \int_{0}^{s} d \tau \hat{F}_{B}(z(\tau))\right)$ with $\hat{F}_{B}$ made of the background field $B_{\mu}$ only.

Analogous expression can be constructed for Green's function of 2-gluon gluelump. It is given by the following expression

$$
\begin{gathered}
G_{\mu v, \lambda \sigma}^{(2 g l)}(x, y)=\left\langle\operatorname { T r } _ { a } \left( f^{a b c} f^{d e f} a_{\mu}^{a}(x) a_{v}^{b}(x) T^{c} \hat{\Phi}(x, y) T^{f} \times\right.\right. \\
\left.\times a_{\lambda}^{d}(y) a_{\sigma}^{e}(y)\right\rangle \equiv N_{c}^{2}\left(N_{c}^{2}-1\right)\left(\delta_{\mu \lambda} \delta_{v \sigma}-\delta_{\mu \sigma} \delta_{v \lambda}\right) G^{(2 g l)}(z) .
\end{gathered}
$$

At small distances $G^{(2 g l)}(x, y)$ is dominated by perturbative expansion terms,

$$
G^{(2 g l)}(z) \sim \frac{1}{z^{4}}
$$

however, as we already discussed in details, all these perturbative terms are canceled by those from higher FCs [12] (triple, quartic, etc...), therefore expansion in fact starts with $n p$ terms of dimension four.

To identify the $n p$ contribution to $G^{(2 g l)}(z)$ we rewrite it as follows:

$$
G^{(2 g l)}(z)=\int_{0}^{\infty} d s_{1} \int_{0}^{\infty} d s_{2}\left(D z_{1}\right)_{0 x}\left(D z_{2}\right)_{0 x} \operatorname{Tr} W_{\Sigma}\left(C_{1}, C_{2}\right)
$$


where the two-gluon gluelump W-loop $W_{\Sigma}\left(C_{1}, C_{2}\right)$ can be written as (in the Gaussian approximation)

$$
\operatorname{Tr} W_{\Sigma}\left(C_{1}, C_{2}\right)=\exp \left\{-\frac{1}{2} \int_{s} \int d \pi_{\mu v}(u) d \pi_{\lambda \sigma}(v)\left\langle\hat{F}_{\mu v}(u) \hat{\Phi} \hat{F}_{\lambda \sigma}(v)\right\rangle\right\}
$$

and the total surface $S$ consists of 3 pieces, as shown in Fig. 1

$$
F_{\mu v} d \pi_{\mu v}(u)=F_{\mu v} d s_{\mu v}(u)-2 i g d \tau(\hat{F}(u))
$$

where $(\hat{F})$ has Lorentz tensor and adjoint color indices $F_{i j}^{a b}$ and lives on gluon trajectories, i.e. on the boundaries of $S_{i}$. In full analogy with (3.5) we have

$$
D(z)=\frac{g^{4}\left(N_{c}^{2}-1\right)}{2} G^{(2 g l)}(z)
$$

The crucial point is that the Green's function (3.6), (3.8) can be calculated in terms of the same FCs $D(z), D_{1}(z)$. Indeed one has

$$
G^{(w)}(z)=\left\langle f\left|\exp \left(-H_{w}|z|\right)\right| i\right\rangle
$$

where the index $w$ stays for 1-gluon or 2-gluon gluelump Hamiltonians. The latter are expressed via the same FCs $D(z), D_{1}(z)$ (see [17] and references therein):

$$
H_{w}=H_{0}[\mu]+\Delta H_{L}[\mu, v]+\Delta H_{\text {Coul }}\left[D_{1}\right]+\Delta H_{\text {string }}[D, v]
$$

where the last term $H_{\text {string }}[D, v]$ depends on $D(z)$ via adjoint string tension

$$
\sigma_{a d j}=\frac{9}{4} \int_{0}^{\infty} d^{2} z D(z)
$$

and Hamiltonian depends on einbein fields $\mu$ and $v$.

The self-consistent regimes correspond to different asymptotics of the solutions to these equations. In Coulomb phase of a gauge theory both $H_{1 g}, H_{2 g}$ exhibit no mass gap, i.e. large $z$ asymptotic of (3.14) is power-like. The function $D(z)$ vanishes in this phase and W-loop obeys perimeter law. In the confinement phase realized in Yang-Mills theory at low temperatures a typical large- $z$ pattern is given by

$$
D(z), D_{1}(z) \sim \exp \left(-|z| / \lambda_{i}\right)
$$

i.e. there is a mass gap for both $H_{1 g}, H_{2 g}$.

Confining solutions are characterized by W-loops obeying area law. In other words, Hamiltonians expressed in terms of interaction kernels depending on $D(z), D_{1}(z)$ exhibit mass gap if these kernels are confining. On the other hand the same mass gap plays a role of inverse correlation length of the vacuum. This should be compared with well known mean-field technique. In our case the role of mean-field is played by quadratic FC, which develops non-trivial Gaussian term $D(z)$. Notice that the exponential form of its large distance asymptotics $\exp (-|z| / \lambda)$ (and not, let's say, $\left.\exp \left(-z^{2} / \lambda^{2}\right)\right)$ is dictated by spectral expansion of the corresponding Green's function at large distances.

Full solution of the above equations is a formidable task not addressed by us here. Instead, as a necessary prerequisite we check below different asymptotic regimes and demonstrate selfconsistency of the whole picture. 
We begin with small distance region. The $n p$ part of contribution to $D_{1}(z)$ at small distances comes from the small distance (small loop) contribution of the Wilson loop with the area $S$ [3], $W(C)=\exp \left(-\frac{\pi^{2}}{8} G_{2} S^{2}\right)$.

As a result one obtains

$$
D_{1}(z)=\frac{4 C_{2}(f) \alpha_{s}}{\pi} \frac{1}{z^{4}}+\frac{g^{2}}{12} G_{2}
$$

It is remarkable that the sign of the $n p$ correction is positive.

At large distances one can use the gluelump Hamiltonian for one-gluon gluelump from [17] to derive the asymptotics

$$
D_{1}(z)=\frac{2 C_{2}(f) \alpha_{s} M_{0}^{(1)} \sigma_{a d j}}{|z|} e^{-M_{0}^{(1)}|z|}, \quad|z| M_{0}^{(1)} \gg 1
$$

where $M_{0}^{(1)}=(1.2 \div 1.4) \mathrm{GeV}$ for $\sigma_{f}=0.18 \mathrm{GeV}^{2}[17,18]$.

We now turn to the FC $D(z)$ as was studied in [16]. The relation (3.13) connects $D(z)$ to the two-gluon gluelump Green's function and we shall write it in the form

$$
G^{(2 g l)}(z)=G_{p}^{(2 g l)}(z)+G_{n p}^{(2 g l)}(z)
$$

where $G_{p}^{(2 g l)}(z)$ contains purely perturbative contributions which are subtracted by higher-order FCs, while $G_{n p}^{(2 g l)}(z)$ contains $n p$ and possible perturbative - $n p$ interference terms. We are interested in the contribution of the FC $\langle F F\rangle$ to $G^{(2 g l)}(z)$, when $z$ tends to zero.

One can envisage two types of contributions:

a) In line with the treatment of $D_{1}$, Eq. (3.17), one can write the term, representing two-gluon gluelump as two nearby one-gluon gluelumps which yields for $D(z)$,

$$
\Delta D_{a}(z)=-\frac{g^{4} N_{c} G_{2}}{4 \pi^{2}}
$$

b) in this case one should consider the diagram given in Fig. 1, which yields the answer (for details see Appendix of [5]).

$$
\Delta D_{b}(z)=2 N_{c}^{2} g^{4} h(z) .
$$

where at small $z, h(z) \approx \frac{D\left(\lambda_{0}\right)}{64 \pi^{4}} \log ^{2}\left(\frac{\lambda_{0} \sqrt{e}}{z}\right), \lambda_{0}$ is of the order of correlation length $\lambda$.

At large distances one use the two-gluon gluelump Hamiltonian as in [17] and find the corresponding spectrum and wave functions, see [17] and appendix 5 of [16] for details. As a result one obtains in this approximation

$$
D(z)=\frac{g^{4}\left(N_{c}^{2}-1\right)}{2} 0.1 \sigma_{f}^{2} e^{-M_{0}^{(2)}|z|}, M_{0}^{(2)}|z| \gg 1
$$

where $M_{0}^{(2)}$ is the lowest two-gluon gluelump mass found in $[17,18]$ to be about $M_{0}^{(2)}=(2.5 \div 2.6)$ $\mathrm{GeV}$.

We shall discuss the resulting properties of $D(x)$ and $D_{1}(x)$ in the next section. 


\section{Discussion of Consistency}

We start with the check consistency for $D(z)$. As is shown above, $D(z)$ has the following behavior at small $z$

$$
D(z) \approx-4 N_{c} \alpha_{s}^{2}(\mu(z)) G_{2}+N_{c}^{2} \frac{\alpha_{s}^{2}(\mu(z))}{2 \pi^{2}} D\left(\lambda_{0}\right) \log ^{2}\left(\frac{\lambda_{0} \sqrt{e}}{z}\right)
$$

Since $\alpha_{s}(\mu(z)) \sim 2 \pi / \beta_{0} \log (\Lambda z)^{-1}$ the first term is subleading at $z \rightarrow 0$ and the last term on the r.h.s. tends to a constant:

$$
D(0)=\frac{N_{c}^{2}}{2 \pi^{2}} D\left(\lambda_{0}\right)\left(\frac{2 \pi}{\beta_{0}}\right)^{2}
$$

Since from (4.2) one can infer, that $D(0) \approx 0.15 D\left(\lambda_{0}\right)$ for $N_{c}=3$, where $\lambda_{0} \gtrsim \lambda^{E}$. So $D(z)$ is an increasing function of $z$ at small $z, z \lesssim \lambda_{0}$, and for $z \gg \lambda$ one observes exponential falloff. The qualitative picture illustrating this solution for $D(z)$ is shown in Fig.2.

This pattern may solve qualitatively the contradiction between the values of $D(0)$ estimated from the string tension $D_{\sigma}(0) \simeq \frac{\sigma}{\pi \lambda^{2}} \approx 0.35 \mathrm{GeV}^{4}$ and the value obtained in naive way from the gluon condensate $D_{G_{2}}(0)=\frac{\pi^{2}}{18} G_{2} \approx(0.007 \div 0.012) \mathrm{GeV}^{4}$. One can see that $D_{\sigma}(0) \approx(30 \div$ 54) $D_{G_{2}}(0)$. This seems to be a reasonable explanation of the mismatch discussed in the introduction.

As was shown in [16], the large distance exponential behavior is selfconsistent, since (assuming that it persists for all $z$, while small $z$ region contributes very little) from the equality $\sigma=\pi \lambda^{2} D_{\sigma}(0)$, comparing with (3.22) one obtains

$$
0.1 \cdot 8 \pi^{2} \alpha_{s}^{2}\left(N_{c}^{2}-1\right) \sigma_{f}^{2}=\frac{\sigma_{f}}{\pi \lambda^{2}}
$$

where in $\alpha_{s}(\mu)$ the scale $\mu$ corresponds roughly to the gluelump average momentum (inverse radius) $\mu_{0} \approx 1 \mathrm{GeV}$. Thus (4.3) yields $\alpha_{s}\left(\mu_{0}\right) \approx 0.4$ which is in reasonable agreement with $\alpha_{s}$ from other systems .

We end this section with discussion of three points:

1) $D(z)$ and $D_{1}(z)$ have been obtained here in the leading approximation, when gluelumps of minimal number of gluons contribute: 2 for $D(z)$ and 1 for $D_{1}(z)$. In the higher orders of $O\left(\alpha_{s}\right)$ one has an expansion of the type

$$
\begin{gathered}
D(z)=D^{(2 g l)}(z)+c_{1} \alpha_{s}^{3} D^{(1 g l)}(z)+c_{2} \alpha_{s}^{3} D^{(3 g l)}(z)+\ldots \\
D_{1}(z)=D^{(1 g l)}(z)+c_{1}^{\prime} \alpha_{s}^{3} D^{(2 g l)}(z)+\ldots
\end{gathered}
$$

Hence the asymptotic behavior for $D(z)$ will contain exponent of $M_{0}^{(1)}|z|$ too, but with a small preexponent coefficient.

2) The behavior of $D(z), D_{1}^{(n p)}(z)$ at small $z$ is defined by NP terms of dimension four, which are condensate $G_{2}$, and the similar term from the expansion of $\exp F$, namely,

$$
\langle\exp F\rangle=1+4 g^{2} \int_{0}^{s} d \tau \int_{0}^{s^{\prime}} d \tau^{\prime}\left\langle F(u(\tau)) F\left(u\left(\tau^{\prime}\right)\right\rangle+\ldots\right.
$$

therefore one does not encounter mixed terms like $O\left(\frac{m^{2}}{z^{2}}\right)$. 
3) correlation lengths $\lambda, \lambda_{1}$ are found from gluelump masses,

$$
\lambda_{1}=1 / M_{0}^{(1)}, M_{0}^{(1)} \approx 1.2 \div 1.4 \mathrm{GeV}, \lambda_{1} \cong 0.2 \div 0.15 \mathrm{fm} .
$$

The value $M_{0}^{(1)}$ in (4.6) is taken from the calculations in [17]. The same is true for $D$, as it is seen from (3.8), where $G^{(2 g l)}$ corresponds to two-gluon subsystem angular momentum $L=0$ independently of $\mu v, \lambda \sigma$.

Hence one obtains

$$
\lambda \equiv \frac{1}{M_{0}^{(2)}} ; \lambda \cong 0.08 \mathrm{fm}, M_{0}^{(2)} \approx 2.5 \mathrm{GeV} .
$$

where the value $M_{0}^{(2)} \approx 2.5 \mathrm{GeV}$ is taken from [17].

\section{Conclusions}

We have derived, following the method of $[13,14]$ the expressions for FC $D(z), D_{1}(z)$ in terms of gluelump Green's functions. This is done in Gaussian approximation. The latter are calculated using Hamiltonian where $n p$ dynamics is given by $D^{n p}(z), D_{1}^{n p}(z)$. In this way one obtains selfcoupled equations for these functions, which allow two types of solutions: 1) $D^{n p}(z)=$ $0, D_{1}^{n p}(z)=0$, i.e. no $n p$ effects at all; 2) $D^{n p}(z), D_{1}^{n p}(z)$ are nonzero and defined by the only scale, which should be given in QCD, e.g. string tension $\sigma$ or $\Lambda_{Q C D}$. All other quantities are defined in terms of these basic ones. We have checked consistency of selfcoupled equations at large and small distances and found that to the order $\mathscr{O}\left(\alpha_{s}\right)$ no mixed perturbative- $n p$ terms appear. The function $D_{1}(z)$ can be represented as a sum of perturbative and $n p$ terms, while $D(z)$ contains only $n p$ contributions.

We have found a possible way to explain the discrepancy between the average values of field strength taken from $G_{2}$ and from $\sigma$ by showing that $D(z)$ has a local minimum at $z=0$ and grows at $z \sim \lambda$. Small value of $\lambda$ and large value of the gluelump mass $M_{g l}=1 / \lambda \approx 2.5 \mathrm{GeV}$ explains the lattice data for $\lambda \approx 0.1 \mathrm{Fm}$. Thus the present paper argues that relevant degrees of freedom ensuring confinement are gluelumps, described self-consistently in the language of FCs.

\section{Acknowledgments}

The work is supported by the grant for support of scientific schools NS-4961.2008.2.

\section{References}

[1] Yu.A.Simonov, Phys. Uspekhi, 39 (1996) 313.

[2] D.S.Kuzmenko, V.I.Shevchenko, Yu.A.Simonov, Phys. Uspekhi, 47 (2004) 1.

[3] H.G.Dosch, Phys. Lett. B 190 (1987) 177; H.G.Dosch and Yu.A.Simonov, Phys. Lett. B 205 (1988) 339; Yu.A.Simonov, Nucl. Phys. B 307 (1988) 512; A.Di. Giacomo, H.G.Dosch, V.I.Shevchenko and Yu. A.Simonov, Phys.Rept., 372 (2002) 319, hep-ph/0007223.

[4] J. Ambjorn and P. Olesen, Nucl. Phys. B 170 (1980) 60.

[5] V.I.Shevchenko, Yu.A.Simonov, arXiv: 0902.1405 
[6] M.A.Shifman, A.I.Vainshtein, V.I.Zakharov, Nucl. Phys. B 147 (1979) 385, 448.

[7] A.V.Nefediev, Yu.A.Simonov and M.A.Trusov, Int. Journ. Mod. Phys. E 18 (2009) 549.

[8] V.I.Shevchenko, Yu.A.Simonov, Phys. Rev. Lett. 85 (2000) 1811; Int. J. Phys. A 18 (2003) 127; G.Bali, Phys. Rev. D 62 (2000) 114503.

[9] A.Di Giacomo and H.Panagopoulos, Phys. Lett. B 285 (1992) 133; M.D’Elia, A.Di Giacomo, and E.Meggiolaro, Phys. Lett. B 408 (1997) 315; A.Di Giacomo, E.Meggiolaro and H.Panagopoulos, Nucl. Phys. B 483 (1997) 371; M.D’Elia, A.Di Giacomo, and E.Meggiolaro, Phys. Rev. D 67 (2003) 114504; G.S.Bali, N.Brambilla and A Vairo, Phys. Lett. B 42 (1998) 265.

[10] Yu.A.Simonov, Yad. Fiz. 54 (1991) 192;

Yu.A.Simonov, in: "QCD: Perturbative or nonperturbative?" eds. L.Ferreira., P.Nogueira, J.I.Silva-Marcos, World Scientific, 2001, hep-ph/9911237.

[11] Yu.A.Simonov, J.A.Tjon, Ann. Phys. 300 (2002) 54.

[12] V.I.Shevchenko, Yu.A.Simonov, Phys. Lett. 437 (1998) 146.

[13] B.S.De Witt, Phys. Rev. 162 (1967) 1195, 1239; J.Honerkamp, Nucl. Phys. B 48 (1972) 269; G.'t Hooft Nucl. Phys. B 62 (1973) 444, Lectures at Karpacz, in : Acta Univ. Wratislaviensis 368 (1976) 345; L.F.Abbot, Nucl. Phys. B 185 (1981) 189

[14] Yu.A.Simonov, Phys. At Nucl. 58 (1995) 107, hep-ph/9311247; Yu.A.Simonov, JETP Lett. 75 (1993) 525; Yu.A.Simonov, in: Lecture Notes in Physics v.479, p. 139; ed. H.Latal and W.Schweiger, Springer, 1996.

[15] Yu.A.Simonov, Phys. Atom. Nucl. 61 (1998) 855, arXiv: hep-ph/9712250.

[16] D.V.Antonov, Yu.A.Simonov, Phys. Atom Nucl. 60 (1997) 478, arXiv:hep-ph/9507254; Yu.A.Simonov, Phys. Atom. Nucl. 61 (1998) 855, arXiv: hep-ph/9712250.

[17] Yu.A.Simonov, Nucl. Phys. B 592 (2001) 350.

[18] M.Foster, C.Michael, Phys. Rev. D 59 (1999) 094509. 


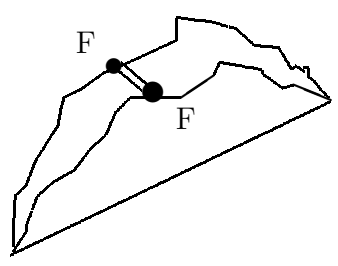

Fig.1

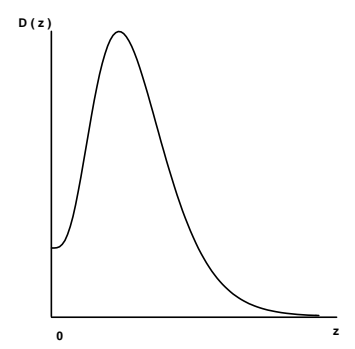

1

Fig. 2 